\title{
Acute Toxicity of Two Tropical Plant Extracts on the Fecundity and Fertility of Culex Quinquefasciatus Say
}

\author{
E. Pushpalatha
}

Department of Zoology, University of Calicut, India

Copyright (C) 2015 Horizon Research Publishing All rights reserved.

\begin{abstract}
Acute toxicity tests were conducted to determine the biological effects of partially purified extract of two tropical plants Croton hirtus L'Her., Pogostemon quadrifolius (Benth.) F.Muell on the juveniles of Culex quinquefasciatus Say. Rearing of C. quinquefasciatus larvae in water at concentration less than the critical concentrations for inhibition of adult emergence in $50 \%$ of treated larvae $\left(\mathrm{EC}_{50}\right)$ of the ethyl acetate fraction of Croton hirtus and Pogostemon quadrifolius leaf extracts from hatching to emergence significantly decreased the fecundity of the $C$. quinquefasciatus and the hatchability of their eggs. At highest concentration of $50 \% \mathrm{EC}_{50}$ of the extracts the decrease in the fecundity over the control ranged between 72.4 and $85.4 \%$.
\end{abstract}

Keywords Plant extracts, Croton Hirtus, Pogostemon Quadrifolius, Culex Quinquefasciatus

\section{Introduction}

In view of the fact that mosquitoes develop genetic resistance to synthetic insecticides [1] and even to biopesticides such as Bacillus sphaericus [2,3] and the application of easily degradable botanicals for the control of mosquitoes is recommended [4]. Alkaloids, nicotine, anabasine, methyl anabasine and lupinine extracted from the Russian weed Anabasis were reported to have high larvicidal activity against Culex spp. [5]. Thangam and Kathiresan [6 and 7] have reported that acetone extracts of several species of marine angiosperm and algae possess very high mosquito larvicidal activity with their critical lethal concentrations $\left(\mathrm{LC}_{50}\right)$ ranging from 17.0 to $95.5 \mathrm{ppm}$. In an effort to increase the activity of plant extracts Pushpalatha and Muthukrishnan [8] purified the crude extracts in silica gel columns using solvents with a sharp polarity gradient and reported that $8.21 \mathrm{ppm}$ of 3:1 petroleum ether (PE): ethyl acetate (EA) fraction of Vitex negundo leaf extract killed $50 \%$ of the treated II instar larvae of Culex quinquefasciatus. As a result of mosquitoes transmitting diseases, the critical concentrations of botanicals which inhibit $50 \%$ of the treated larval population from emerging as adults (EC50) are more meaningful than the LC50 $[9,10]$. The larvicidal activity of extracts from two tropical plants and the effect on fecundity and fertility against $C$. quinquefasciatus are reported in this paper.

\section{Materials and Methods}

\section{i) Preparation of Plant extracts}

Mature leaves of Croton hirtus and Pogostemon quadrifolius were collected from the Calicut University Campus, dried under shade, powdered and extracted with analytical grade methanol $(\mathrm{MeOH})$. The $\mathrm{MeOH}$ extract was concentrated and defatted with equal volume of $\mathrm{MeOH}$ and Petroleum ether (PE). The defatted $\mathrm{MeOH}$ fraction was then fractionated into ethyl acetate (EA) and water soluble fractions (Alkofahi et al [4]; modified). Preliminary bioassay tests revealed that the EA fractions of Croton hirtus and Pogostemon quadrifolius leaf extract were more toxic than the other fractions hence only these fractions were selected to study their effect on the fertility of the mosquitoes. The most active fractions of the selected plants were prepared and stored in refrigerator at $15^{\circ} \mathrm{C}$.

\section{ii) Bioassay (Estimation of LC50 and EC50)}

Larvae of C. quinquefasciatus used in the present study were obtained from the laboratory culture maintained as described in Pushpalatha and Muthukrishnan [8]. Freshly hatched or moulted larvae were used for the bioassays. Different concentrations of the various fractions of the selected plant extracts were prepared in glass bowls. 20 freshly hatched /moulted larvae (I-IV instar) were exposed to each concentration in triplicates. Two different controls (water without methanol and water with the maximum volume of methanol used for making up the desired concentrations of the test medium) were maintained for each treatment. Mortality of the treated and control larvae over a period of $24 \mathrm{hr}$ was observed and percent mortality was calculated for the estimation of LC50 using a modified probit 
programme by Finney [11].

Freshly hatched first instar larvae were reared until their emergence as adults, in water with different concentrations less than the effective critical concentrations of the most active fractions of the extracts for inhibition of emergence (EC50) of $50 \%$ of the treated larvae. To $100 \mathrm{ml}$ of water taken in sterilized glass bowls the appropriate volume of $1 \%$ stock solution of the most active fractions of the selected plant extracts were added to obtain 5, 10, 25 and $50 \%$ of the EC50 of the extracts in the medium. The control medium $(0 \%$ EC50) contained the maximum volume of $\mathrm{MeOH}$ in the test medium. Fifty freshly hatched C. quinquefasciatus larvae were introduced into the different bowls. Three replicates were maintained for each tested concentration. The larvae provided with powdered dry yeast and dog biscuits in the ratio of 3:1. The level of water in the bowls was maintained by adding the required volume of dechlorinated water. After the larvae metamorphosed into pupae they were transferred to emergence cages and allowed to emerge. With the help of an aspirator 10 males and 10 females that emerged from each treatment were introduced into oviposition cages. The males were provided with $10 \%$ sugar solution through cotton buds the females with a blood meal from an immobilized chicken kept overnight inside the cage. The oviposition cages were covered with a wet cloth to maintain constant humidity $(85 \pm 5 \%)$. One bowl containing dechlorinated water was also kept inside the cage to facilitate oviposition by females. The egg rafts oviposited by the females were removed from the cage next morning, counted under the low power of a binocular microscope and allowed to hatch. Fecundity (number of eggs/female) was monitored until the females died. The number of eggs that hatched into first instar larvae in each concentration was counted and hatchability was calculated as percentage of eggs deposited. Following Saxena et al [12], the sterility index (SI) was calculated as follows SI $=100$ - $\{$ Fecundity of the treated larvae $\mathrm{X}$ hatchability $(\%) /$ fecundity of the control X hatchability $\%$ \}
X 100.

\section{Results}

Fig 1and 2 presents $24 \mathrm{hr} \mathrm{LC}_{50}$ (ppm) of the different fractions of the selected plant extracts for the different instars of $C$. quinquefasciatus larvae. The result show that EA fraction of the Croton hirtus and Pogostemon quadrifolius leaf extracts were the most active of the different fractions obtained. Table 1 provides data on $\mathrm{EC}_{50}(\mathrm{ppm})$ and $\mathrm{EC}_{90}$ (ppm) of the EA fractions of the selected plant extracts. The EA fraction of Croton hirtus and Pogostemon quadrifolius leaf extract was very highly active with regard to its efficacy to inhibit adult emergence The lowest $\mathrm{EC}_{50}(\mathrm{ppm})$ of the EA fraction is ranged between $8.79 \mathrm{ppm}$ and $19.1 \mathrm{ppm}$ against II instar larvae for Croton hirtus and Pogostemon quadrifolius respectively.

From the data provided in table 2 a dose dependent decrease in the fecundity of the selected species of mosquito obtained from the larvae subjected to treatment with less than $\mathrm{EC}_{50}$ of the different extracts from hatching to emergence is evident. C. quinquefasciatus females in the control series deposited 136.4-140.9 eggs. Treatment of $C$. quinquefasciatus with $50 \%$ of the $\mathrm{EC}_{50}$ of I instar Culex of $C$. hirtus leaf extract decreased the fecundity by $85.4 \%$. On the other hand treatment of $C x$. quinquefasciatus with $50 \%$ of $\mathrm{EC}_{50} \quad$ I instar Culex of $P$. quadrifolius leaf extract decreased the fecundity by $72.39 \%$ over control. Treatment of larvae, especially with $50 \%$ of the $\mathrm{EC}_{50}$ of the active fractions of the extracts significantly decreased the hatchability of the eggs oviposited by the adults obtained from them (table 2). Treatment of the larvae with $50 \%$ of the $\mathrm{EC}_{50}$ of the extracts induced 86 to $91 \%$ sterility in the progeny of $C$. quinquefasciatus. Briefly, the active fraction of the two selected plant extracts decreased the fertility of the $C$. quinquefasciatus significantly.

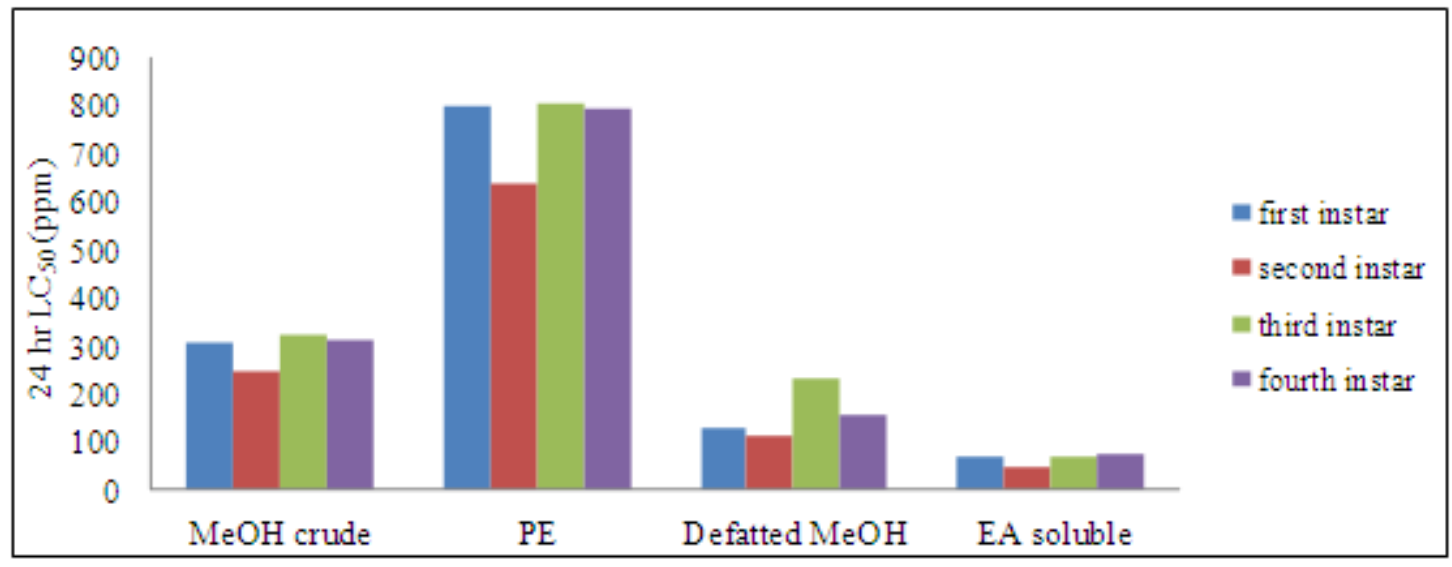

Figure 1. $24 \mathrm{hr} \mathrm{LC}_{50}(\mathrm{ppm})$ of the different fractions of $C$. hirtus leaf extracts on the different instars of Cx. quinquefasciatus. 


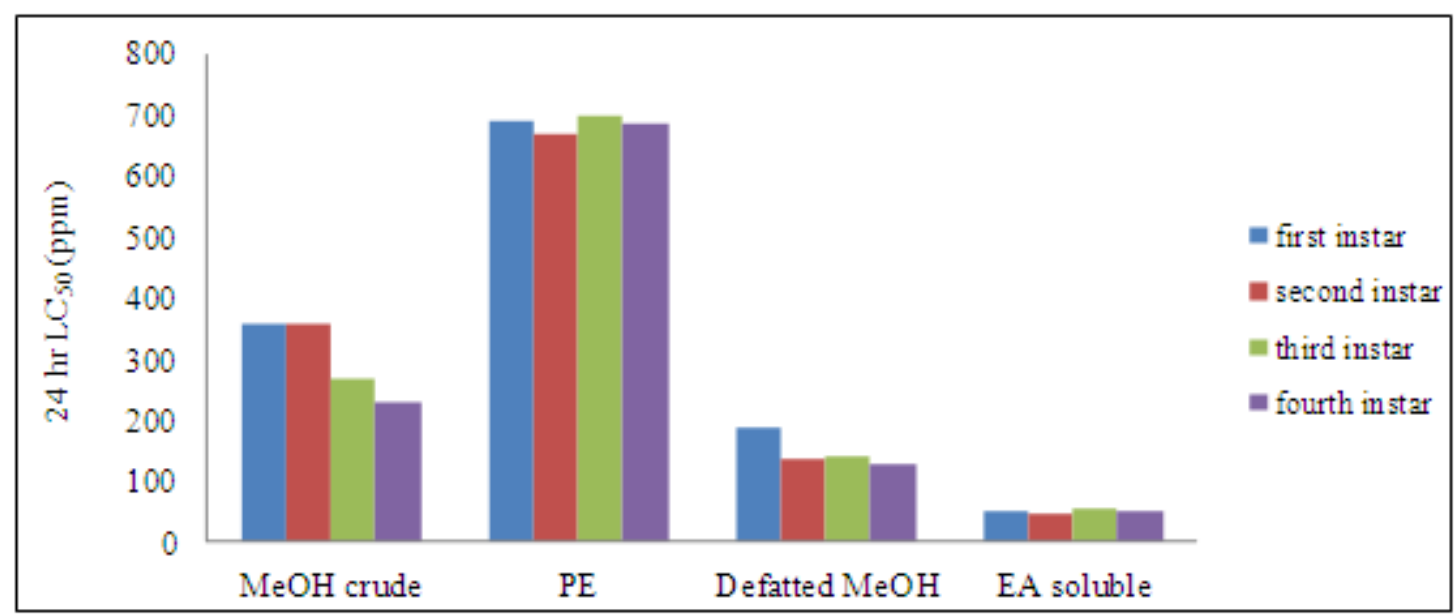

Figure 2. $24 \mathrm{hr} \mathrm{LC}_{50}$ (ppm) of the different fractions of P. qudrifolius leaf extracts on the different instars of Cx. quinquefasciatus.

Table 1. $\mathrm{EC}_{50}(\mathrm{Mean} \pm \mathrm{SI}) \mathrm{ppm}$ of the $\mathrm{EA}$ fractions of the selected plant extracts tested against different instars of $\mathrm{C} x$. quinquefasciatus

\begin{tabular}{|c|c|c|c|}
\hline Plant & Instar & EC50 (ppm) & EC90 (ppm) \\
\hline \multirow{4}{*}{ C. hirtus } & I & $10.85 \pm 0.63$ & $93.7 \pm 1.12$ \\
\cline { 2 - 4 } & II & $8.79 \pm 0.37$ & $65.0 \pm 1.48$ \\
\cline { 2 - 4 } & III & $9.46 \pm 0.44$ & $74.3 \pm 1.28$ \\
\cline { 2 - 4 } & IV & $12.93 \pm 0.72$ & $82.8 \pm 1.07$ \\
\hline \multirow{4}{*}{ P.quadrifolius } & I & $23.9 \pm 1.34$ & $98.3 \pm 12.7$ \\
\cline { 2 - 4 } & II & $19.1 \pm 1.24$ & $84.9 \pm 6.37$ \\
\cline { 2 - 4 } & III & $28.9 \pm 1.49$ & $129.7 \pm 9.21$ \\
\cline { 2 - 4 } & IV & $25.8 \pm 1.83$ & $124.1 \pm 9.36$ \\
\hline
\end{tabular}

Table 2. Effect of EA fraction of the selected plant extracts on fecundity and hatchability of the egg of $C x$. quinquefasciatus. Each value represents the mean $\pm \mathrm{SD}$ of three replicates of ten females raised from the treatment of sub lethal concentrations, subjected to ad libitum mating.

\begin{tabular}{|c|c|c|c|c|c|c|}
\hline Plant & $\begin{array}{c}\text { Conc, } \\
\text { (ppm) }\end{array}$ & $\begin{array}{c}\text { Fecundity } \\
(\mathrm{egg} / \text { female })\end{array}$ & $\begin{array}{c}\text { Hatchability } \\
(\%)\end{array}$ & $\begin{array}{c}\text { Decrease over control Fecundity } \\
\text { Hatchability }\end{array}$ & Sterility Index \\
\hline \multirow{4}{*}{ C. hirtus } & Control & $136.4 \pm 0.80$ & $83.0 \pm 0.5$ & 0 & 0 & -- \\
\cline { 2 - 7 } & 5 & $98.8 \pm 0.68$ & $82.2 \pm 0.5$ & 27.4 & 1.0 & 29.45 \\
\cline { 2 - 7 } & 10 & $63.6 \pm 0.87$ & $69.3 \pm 1.2$ & 53.4 & 16.5 & 61.15 \\
\cline { 2 - 7 } & 25 & $44.3 \pm 2.21$ & $64.6 \pm 3.1$ & 67.5 & 22.5 & 74.37 \\
\cline { 2 - 7 } & 50 & $19.97 \pm 8.33$ & $49.8 \pm 2.78$ & 85.4 & 40.0 & 91.49 \\
\hline \multirow{5}{*}{ P.quadrifolius } & Control & $140.9 \pm 0.81$ & $97.9 \pm 0.2$ & 0 & 0 & -- \\
\cline { 2 - 7 } & 5 & $136.2 \pm 0.44$ & $74.1 \pm 1.98$ & 3.34 & 24.3 & 26.9 \\
\cline { 2 - 7 } & 10 & $96.1 \pm 0.70$ & $73.0 \pm 0.62$ & 31.8 & 25.4 & 49.2 \\
\cline { 2 - 7 } & 25 & $68.6 \pm 0.21$ & $60.1 \pm 0.81$ & 51.32 & 38.6 & 70.2 \\
\cline { 2 - 7 } & 50 & $34.9 \pm 1.01$ & $49.8 \pm 0.12$ & 72.39 & 49.1 & 86.0 \\
\hline
\end{tabular}

\section{Discussion}

The plants tested in the present study are reported to have medicinal value and are not toxic to vertebrates [13]. Observations on critical concentrations have shown that the critical lethal concentration $\left(\mathrm{LC}_{50}\right)$ and the $\mathrm{EC}_{50}$ of these extracts are far less than other partially purified extracts and that these extracts are highly efficacious for the control of mosquitoes. Results on the sterilizing effects of the different concentrations less than $\mathrm{EC}_{50}$ of the active fraction of the selected plant extracts on C. quinquefasciatus reported in the present study confirm their potential for control of mosquito population. The larvae which survive the treatment with sub lethal concentrations manage to emerge as adult ultimately oviposit a very few eggs, most of which fail to hatch successfully. The negative effects of these extracts on the fecundity and hatchability of eggs of the mosquitoes are remarkably greater than those reported for other plant extracts in the literature $[10,12)$. The effect of the extracts on the fecundity and hatchability reported in the present study 
prompt to speculate that the extracts produce these effects through their influence on the endocrine system. However further studies on the mechanism of action of these extracts and their efficacy for control is required for the development of a more potent bio control agent for the control of mosquito vectors.

\section{REFERENCES}

[1] Wattal, B.L.,Joshi,G.C., and Das, M.(1981). Roll of agricultural insecticides in precipitating Vector resistance. J. Comm. Dis. 13, 71-73

[2] Tabashnik, B.E. (1994). Evolution of resistance to Bacillus thuringiensis. Annu. Rev. Entomol, 39, 47-79

[3] Rodcharoen, J. and Mulla, M.S., (1994). Resistance development in Culex quinquefasciatus (Diptera: Culicidae) to the microbial agent Bacillus sphaericus. J. Econ. Entomol, $87,1133-1140$

[4] Alkofahi, H., Rupprecht, J. K., Anderson, J. E., McLaghlin, J.L., Mikolajczak, K.L. and Scott, B. A. (1989). Search for new pesticides from higher plants. In: Insecticides of Plant Origin. Ed. By Arnason, J.T., Philogene, B.J.R., Morand P. Washington: Amer. Chem. Society. Pp. 25-43

[5] Campbell, F.L., Sullivan, W.W. and Smith, L, N. (1993). The relative toxicity of nicotine, anabasine, methyl anabasine and lupinine for culicine mosquito larvae. J.Econ.Entomol. 26,
500-509.

[6] Thangam, T.S and Kathiresan, K. (1988a) Toxic effect of mangrove plant extracts on mosquito larvae, Anopheles stephensi. Curr. Sci. 57, 914-915.

[7] Thangam, T.S and Kathiresan, K. (1988,b). Toxic extracts of sea weed extracts on mosquito larvae. J. Med.Res. 88, $35-37$

[8] Pushpalatha E.and Muthukrishnan, J (1995). Larvicidal activity of a few plant extracts against Culex quinquefasciatus and Anopheles stephensi. Indian J. Malariol.32, 14-23

[9] Baktharatchagan, R., Rita, C. and Jebanesan A (1993). Laboratory evaluation of two insect growth regulators against some vector mosquitoes. J. Insect Sci,6, 276-278

[10] Mohsen, Z.H, Jawad,A.M., Al-A=Saadi, M, and Al-Naib B.A.(1995). Anti-oviposition and insecticidal activity of Imperata cylindrical (Graminae). Med, Vet. Entomol. 9, 441-442

[11] Finney, D.J.(1971). Probit analysis $3^{\text {rd }}$ edn. Cambridge: Cambridge University Press.p.333

[12] Saxena, R.C., Hrashan, V., Saxena, A. and Sukumaran, P. (1993). Larvicidal and chemosterillant activity of Annona squamosa alkaloids against Anopheles stephensi. J.Amer. Mosq. Control Assoc. 9, 84-87

[13] Chopra, R.N., Nayar, S.L., and Chopra, I.C.(1956). Useful plants of India. New Delhi: C.S.I.R. p. 178 